\title{
The five 'C's': synergies in International Disaster Management and public health and a place for entrepreneurial resilience?
}

There is a growing awareness of the requirement to reconcile disaster management and public health priorities over the coming decade. Lee Miles, Professor of Crisis and Disaster Management at Bournemouth University Disaster Management Centre, outlines the five main challenges involved to integrate public health into any effective disaster management system.

\section{INTRODUCTION: TRAJECTORIES} OF SENDAI AND BEYOND

The Sendai Framework for Disaster Risk Reduction, that sets out the international agenda for disaster management from 2015 to 2030, has already identified the pressing need for cooperation in advancing public health resilience. ${ }^{1}$ Most notably, the Sendai Framework provides for numerous voluntary commitments with a specific public health focus, including (1) enhancing the resilience of national health systems through training and capacity development and integrating disaster management into primary, secondary and tertiary health care (p. 18; also see later); (2) strengthening the design and implementation of inclusive policies and social safety-nets (p. 19), such as access to basic health services; and (3) finding robust post-disaster solutions that will assist people most directly affected by disasters, including those with life threatening and chronic disease (p. 20). ${ }^{2}$ Indeed, work has already begun to put flesh on these bones. On 10 March 2016, the United Nations (UN) Office for Disaster Risk Reduction and the World Health Organization jointly issued an appeal for national disaster management agencies to develop their preparedness/ response capacities to go beyond natural disasters like earthquakes to include health emergencies, highlighting how recent events like the Ebola epidemic and the Zika virus outbreak underline the need to 'break down the silos' between disaster managers and health workers and increased readiness for deployment in public health emergencies, where the trigger may be a virus like Zika.

This trajectory has similarly not gone unnoticed among the UK public health authorities. Building upon the UK government's post-2008 strategy, recognising that 'Health is Global' 3 and that natural catastrophes and man-made disasters 'endanger the collective health of populations across geographical regions and international boundaries', Public Health England's (PHE) Global Health Strategy 2014-20194 envisages the development of 'a cadre of trained staff ... to build public health capacity and resilience for the longer term'.

\section{CONSIDERING THE FIVE 'C'S'}

Future trajectories then envisage closer synergising of disaster management and public health agendas and capacities. Yet, if this is the case, it is also necessary to have a nuanced understanding of the nature of challenges ahead, and what such synergising will need to cope with. Briefly, this article identifies these challenges as the five 'C's'. These are as follows:

- C1: Challenge of Consistency. For a long time, health parameters, like numbers of fatalities and size of casualties, have been an integral and rather consistent measurement of the seriousness, magnitude, scale and impact of disasters - and equally important in communicating the importance, proportionality and even effectiveness of response and recovery efforts in terms of lives saved and casualties rescued and rehabilitated. Moreover, with climatic change affecting an increasingly interdependent world, parts of the globe are not just experiencing regularity of the disasters but also the emergence of new public health threats that have avenues for international transmission. In a cumulative sense, the outcome is that the expectations of, and demands placed on, public health systems and services are higher and constant, and where synergising should be 'business as usual'.

- C2: Challenge of Cascading. Recently, it has been popular to identify the nature of 'cascading disasters', 5 such as the 2012 Fukushima disaster, which unfolded over time and presented evolving public health issues. Cascading assumes that, like toppling dominoes, there are chain-sequences of cause and effect, with disasters over time that lead to catastrophic outcomes and progressively larger, but also changing challenges for public health over the short and long term. By identifying how disaster are likely to cascade, public health systems are able to theoretically assess and plan more effectively to cope with potential and proportionate cascading public health risks over time.

- C3: Challenge of Complexity. Alongside cascading, there also needs to be recognition that disasters are also incredibly complex events that have complicated and simultaneous political, economic, social and health implications not just over 'time' (cascading) but also over 'space' (complexity). Given the 
panoramic effect of disasters, public health issues will feature in most spheres of disaster activity requiring public health officials to play wider roles than normal and placing multiple and sometimes contradictory demands that public health services will often find challenging to manage effectively. As the recent Ebola and Zika outbreaks illustrate, global interdependency also increases the complexity of threats that public health agencies have to address. In many ways, it should be expected that public health systems will find it difficult to grapple with the unexpected complexity of the 'unknown, unknowns' of disaster as they unfold.

- C4: Challenge of Connectivity. The challenging cascading and complexity of disasters will require the public health system to build new networks and enhance effective coordination both horizontally among public health stakeholders and vertically across the regional, national and international levels of disaster management to ensure they are 'connected'. In terms of public health, this implies that public health planning and provision needs to be integrated across levels, sectors, services and even borders to handle the international nature of disasters, as Ebola experience illustrates.

- C5: Challenge of Capacity. Disasters have panoramic, comprehensive connotations for public health provision. The Sendai Framework objectives, for example, include commitments to reducing mortality, improving casualty tracking, enhanced involvement of stakeholders and improved access to health services - which should be accommodated into short-term and long-term capacity-building of public provision for use both home and abroad, and in regional, national and international settings. In simple terms, perceptions are that disasters today have such magnitude and happen with growing regularity, that it is unwise to treat them as ad hoc and/or exceptional 'items' for public health systems or, indeed, for those public health bodies committed to international relief operations abroad. Synergising assumes public health capacity-building.

\section{PUBLIC HEALTH REALITY: THE CENTRALITY OF INTEGRATED TRAINING}

The implications of the five 'C's' are notable. As a bare minimum, it is essential for public health objectives and representation to be further incorporated into disaster management planning since public health questions, issues, priorities and capacities are never far below the surface in terms of affecting all aspects of the disaster management cycle - from mitigation, preparedness and response to recovery phases. Public health questions, and indeed healthy populations, are often central to securing more resilient societies that are able to handle disaster quickly, appropriately and consistently. Yet, if public health is to be fully integrated into any effective disaster management system, this requires public health actors to be willing to be integrated and participate. It is partly about mind-sets. Equally, disaster management priorities and the pursuit of organisational, community and even individual resilience must feature strongly within logics of public health planning so that disaster management is accepted as an integrated part of public health provision. Integration within and between disaster management and public health is therefore key.

Turning to aforementioned priorities of Sendai, one aspect is worthy of further consideration, namely, the importance of training. It is imperative that public health concerns are incorporated into situational awareness, risk analysis and assessment, and casualty tracking aspects of the training of disaster managers as a bare minimum, and equally, that disaster management training features strongly in the training of public health professionals in the pursuit of increased public health resilience. An important gap here needs to be closed.

\section{LOOKING TO THE FUTURE:} ENTREPRENEURIAL RESILIENCE In theory, this sounds an easy task. Yet, managing disasters and addressing public health concerns are also both dynamic (and expensive) pursuits and ones where threat perceptions change. Indeed, disaster management is often preoccupied with 'managing expectations' of what can realistically be done to handle the unexpected. Put simply, making abnormal disasters seem as normal as possible and part of the art of the doable. However, as Alexander 6 argues, emergency plans are closer to being skeleton guidelines than future proof disaster management bibles for officials in most instances. In practice, disaster managers and public health officials - as human capacity - are often 'innovating' in terms of policy, tweaking and adapting planning during the onset of crises to handle the unforeseen cascading, complexity and connectivity of disasters. ${ }^{7,8}$

It is advisable then that such figures acquire an extensive, dynamic set of skills and knowledge so that they can be agile and adaptive in disaster situations ${ }^{7,8}$ within the broader parameters of public health. After all, public health responses during emergencies are often based on judgements and actions taken on the basis of imperfect information and best guess estimates about the future in high-intensity environments. This is what this author has labelled elsewhere as developing a capacity for 'entrepreneurial resilience'; where public health officials undertaking disaster management have broader 'soft' skill sets to meet entrepreneurially the dynamic challenges of disasters as they unfold. Hence, if synergising of disaster management and public health is to be effective, it is not just about what PHE have called a commitment to 'co-development', where everyone has something to learn and something to teach (p. 19). ${ }^{4}$ There is also a need to understand and train 'policy entrepreneurs', 7,8 who can innovate and fill in the gaps when disaster planning fail or need to be agile to account for changing public health 
issues. Training of public health officials needs to further synergise with the training of skill sets of disaster managers and to clarify what they jointly require staff to possess, to enhance public health resilience. By focusing on synergised training, trust and confidence will be built facilitating integration and innovation between, on one side, public health resilient disaster managers and, on the other, more disaster aware public health officials to meet the challenges of consistent, cascading, complex and connected public health emergencies in a changing world.

Lee Miles

Disaster Management Centre, Bournemouth University, Bournemouth, UK

\section{[AQ: 1]}

1. Aitsi-Selma A, Egawi S, Sasaki H, Wannous C, Murray V. The Sendai Framework for Disaster Risk Reduction: Renewing the Global Commitment to People's resilience, health and Well-being. International Journal of Disaster Risk Science 2015; 6(2): 164-76.

2. United Nations Office for Disaster Risk Reduction (UNISDR). Sendai Framework for Disaster Risk Reduction 2015-2030. Geneva: UNISDR, 2015.
3. HM Government. Health is Global: An Outcomes Framework for Global Health 2011-2015. London: Department of Health, 2011.

4. Public Health England (PHE). Global Health Strategy 2014 to 2019. London: PHE, 2014.

5. Percaroli G, Alexander D. Critical infrastructure, Panarchies and the vulnerability paths of cascading disasters. Natural Disasters 2016; 82(1): 175-92.
6. Alexander D. How to Write an Emergency Plan. Edinburgh: Dunedin Academic Press, 2016.

7. Miles L, Petridou E. Entrepreneurial resilience: Role of policy entrepreneurship in the political perspective of crisis management. In: Bhamra R (ed.) Organisational Resilience: Concepts, Integration and Practice. Boca Raton, FL: CRC Press, 2015, pp. 67-83.

8. Miles L. Entrepreneurial resilience. Crisis Response Journal 2016; 11(8). [AQ: 2] 